\title{
L'innovation en autobiographie selon Jung : une exploration du média de la bande dessinée
}

\section{Kevin Pat Fong, Université Queen's}

Depuis le succès phénoménal de Maus d'Art Spiegelman ${ }^{1}$, la bande dessinée a obtenu une certaine reconnaissance dans l'institution littéraire. Longtemps reléguée au rang de divertissement, la bande dessinée s'impose désormais comme un médium légitime, partageant un statut similaire aux cuvres venant des médias traditionnels. D'ailleurs, la bande dessinée apporte une certaine fraîcheur en offrant une multitude de techniques narratives et visuelles aux écrivains, qui leur permet de renouveler le canon littéraire ainsi que de présenter de nouveaux enjeux thématiques et stylistiques.

$\mathrm{Jung}^{2}$, scénariste et dessinateur belge d'origine coréenne, partage avec nous son récit autobiographique en noir et blanc intitulé Couleur de Peau : Miel - Tome 1 (1997). Son histoire débute par le récit de son enfance comme orphelin grandissant en Corée du sud, pour être ensuite adopté par une famille belge jusqu'à l'adolescence. Avec un mélange ingénieux d'humour, de sarcasme et de drame, Jung aborde des sujets sérieux et contemporains tels que la quête d'identité, le déracinement, l'abandon, l'intégration et un sujet peu exploré et méconnu du public : l'adoption des enfants coréens par les familles des pays développés.

En tentant de répondre à sa propre question, «Peut-on innover en autobiographie?», Philippe Lejeune est d'avis que les changements dans le genre autobiographique sont influencés par les différents types de représentation disponibles :

Un genre littéraire est une catégorie en mouvement combinant une force d'inertie et de répétition (modèles canoniques, horizons d'attente, que le définisseur tente de fixer...) et une force de changement: la pratique autobiographique s'inspire de l'ensemble des autres pratiques textuelles, et elle est liée à l'évolution de la civilisation sous ses aspects les plus divers : les mœurs, les relations sociales, les sciences de l'homme, les médias, par exemple. (73)

Ainsi, selon Lejeune, la bande dessinée, en référence à Maus, pourrait être considérée comme candidate potentielle. Cela dit, en ce qui concerne Jung et sa bande dessinée, l'auteur réussit-t-il à renouveler le genre autobiographique ? Parvient-il à 
utiliser la bande dessinée pour affirmer son identité ? Quels sont les effets de l'œuvre du dessinateur sur la mémoire individuelle et collective ?

\section{Le média de la bande dessinée}

Par rapport à la question de la représentation, le média de la bande dessinée dispose d'un arsenal impressionnant de techniques et regorge de possibilités.

D'après Will Eisner ${ }^{3}$ dans Comics and Sequential Art (2008), la bande dessinée est un art séquentiel, c'est-à-dire un art qui emploie la juxtaposition des images en séquence mais pas en mouvement. L'art séquentiel requiert qu'un individu prenne en considération le texte et l'image dans une case ainsi que sa connexion avec la prochaine case.

Contrairement au cinéma, la bande dessinée interpose toutes les cases sur une planche (ou une page complète), ce qui permet aux lecteurs de voir non seulement la suite du récit mais aussi de revenir en arrière, de mettre en place un rythme personnel, ce qui affecte effectivement son expérience de l'œuvre. En somme, chaque bande dessinée possède trois niveaux de lecture et de compréhension : le texte, l'image et la séquence.

\section{Le critère imitatif et imaginatif de la bande dessinée}

La bande dessinée offre un terrain fertile à l'imagination des bédéistes, qui peuvent ainsi prendre distance avec la réalité. Dans une tentative de justifier la complexité du média et de le valoriser, Antonio Altariba dans Propositions pour une analyse spécifique de récit en bande dessinée, propose d'étudier le récit en bande dessinée à partir du critère imitatif - lorsqu'il s'agit de copier, et du critère imaginatif lorsqu'il s'agit d'inventer (28).

Le critère imaginatif englobe tous les éléments imaginés par le dessinateur qui désobéissent aux lois de la réalité dans le but d'accentuer un effet, par exemple la satire et le comique, par exemple dans la vignette où Jung est transporté vers l'Europe par une cigogne, ce qui fait allusion au lien imaginaire que partagent l'oiseau symbolique et la livraison des bébés aux familles. De plus, le choix de la cigogne représente non une naissance mais une renaissance comme dans le cas de l'enfant adopté qui se voit offrir une chance de renaître (20). Par opposition, les éléments d'imitation fournis par le 
dessinateur ajoutent un effet d'authenticité. Les illustrations les plus frappantes se manifestent sous des vignettes accentuant la fidélité aux détails réels tels que le document d'adoption de Jung - une imitation dessinée à la main - et les costumes des danseuses traditionnelles coréennes.

À partir de ces deux critères de base, Altariba suggère d'étudier l'art séquentiel à travers trois perspectives : les formes, les fonctions et les articulations. La forme d'une vignette se réfère à l'analyse de la relation entre le personnage ou l'objet principal avec l'arrière-plan et la stylisation. Pour emprunter aux études linguistiques, l'étude de la forme se rapproche à la morphologie. La fonction comprend l'analyse des cadres et les points de vue choisis pour représenter une scène, car dans la plupart des bandes dessinées, la taille et l'angle des cadres varient selon l'effet que veut créer le bédéiste. Finalement, l'articulation prend en compte les relations entre vignettes et la manière où une scène d'action s'enchaîne. L'exemple classique survient quand une case illustre l'acte de donner un coup de poing ; la case suivante montrera alors la conséquence.

\section{L'innovation en autobiographie}

Couleur de Peau: Miel apporte un certain degré d'innovation au genre autobiographique par l'intermédiaire de diverses techniques et modalités de représentation. Commençons par les normes de l'autobiographie en reprenant les travaux de Lejeune. Nous dirions, en effet, que le pacte autobiographique est conclu dès les premières pages du premier chapitre avec l'utilisation du «Je » pour introduire le récit, mais surtout avec l'imitation du document d'adoption dessiné par Jung. 


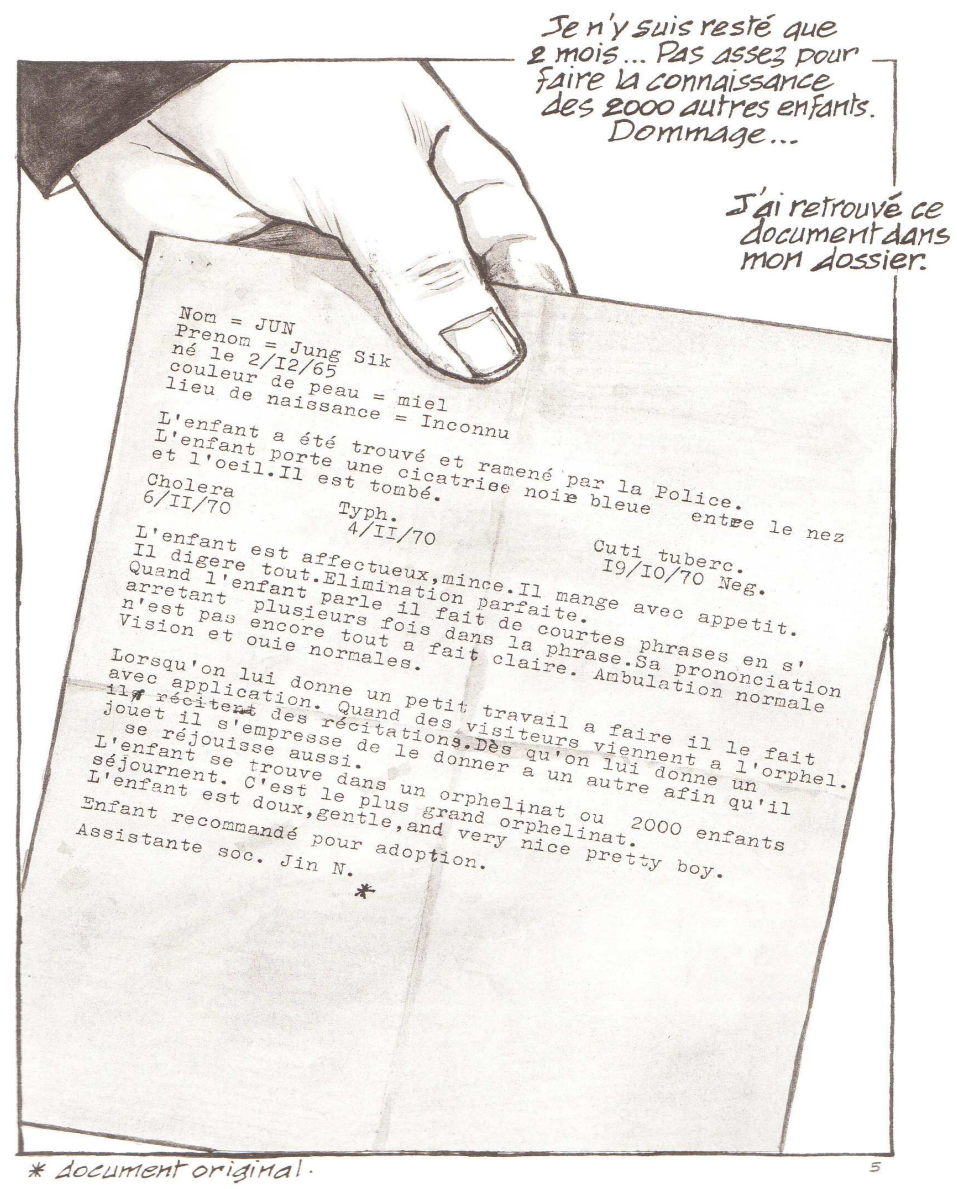

(C) MC Productions / Quadrants / Jung

L'effet d'authenticité est renforcé par la fonction du cadre (point de vue de la représentation). Avec une focalisation zéro et une main tenant le document, nous avons l'impression que la main appartient à l'auteur qui nous présente directement le document en question, surtout avec le choix de montrer la feuille dans son intégralité, ce qui a pour effet de couvrir le panneau ${ }^{4}$ en entier. Pour en revenir à la forme (relation entre personnage et arrière-plan) de cette page, la stylisation reste très fidèle à l'imitation par le contenu tapé à la machine. Ironiquement, Jung insiste, grâce à cette note au bas de page, sur l'originalité du document, un choix esthétique qui pourrait être interprété comme un effort de préserver le ton comique de la bande dessinée ou peut-être pour sceller le pacte autobiographique avec les lecteurs.

En ce qui concerne le pacte autobiographique, nous nous trouvons en la possession d'un paratexte très bien documenté avec l'inclusion de photos authentiques du bédéiste quand il était enfant avec son numéro d'identité inscrit sur son pancarte, un aspect autobiographique qu'il reproduit dans une des cases du premier chapitre. 

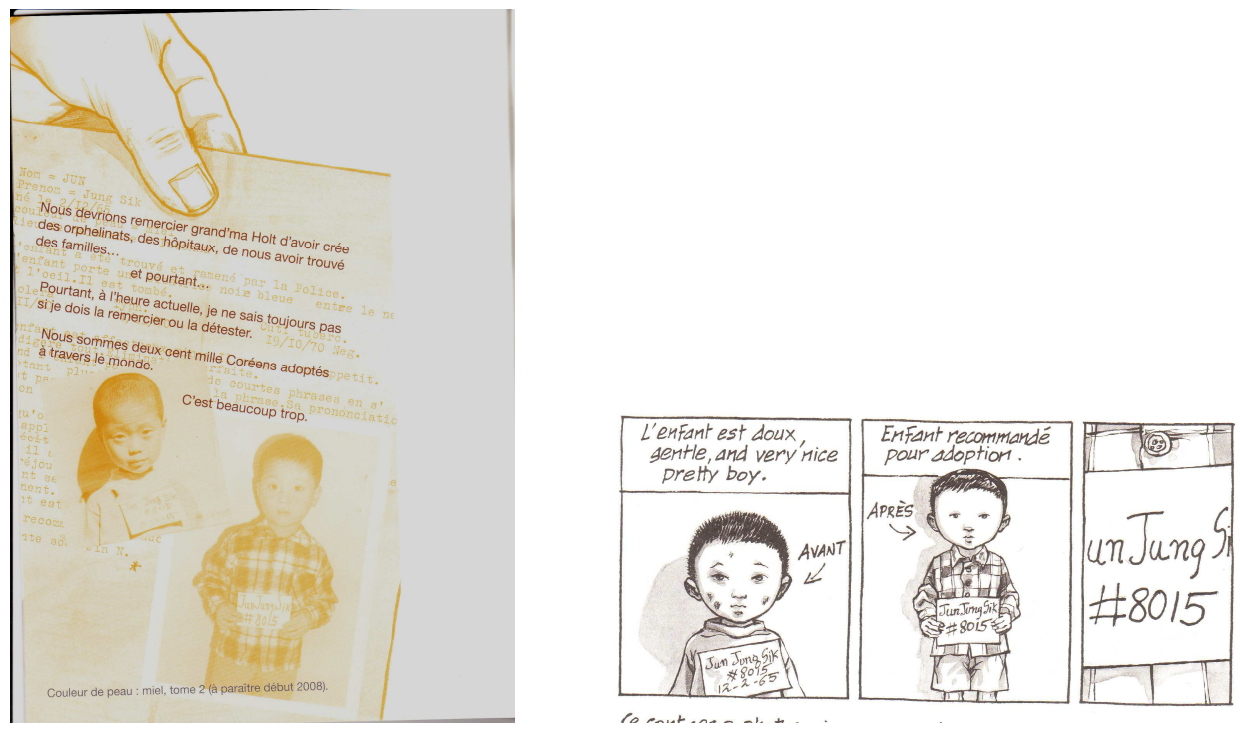

MC Productions / Quadrants / Jung

Cette décision d'intégrer des photos personnelles influence sans aucun doute la réception de l'œuvre. Lejeune reconnaît l'importance du paratexte et de sa fonction dans la consolidation du pacte autobiographique :

L'identité de nom entre auteur, narrateur et personnage peut être établie de deux manières :

1. Implicitement, au niveau de la liaison auteur-narrateur, à l'occasion du pacte autobiographique ; [...]

2. De manière patente, au niveau du nom que se donne le narrateurpersonnage dans le récit lui-même, et qui est le même que celui de l'auteur sur la couverture.

Il est nécessaire que l'identité soit établie au moins par l'un de ces deux moyens ; il arrive souvent qu'elle le soit par les deux à la fois. (27)

Il faut souligner que Lejeune se réfère à des formes traditionnelles. Mais est-ce que la bande dessinée arrive à renouveler son utilisation ? En effet, les photos authentiques prouvent la validité du numéro d'adoption de Jung. Mais nous voyons ici que Jung s'illustre différemment dans le sens qu'il ne produit pas une imitation de son portrait réel. Il choisit de se représenter en personnage de bande dessinée pour se conformer aux normes stylistiques de son œuvre. S'agit-il alors d'une imitation du pacte autobiographique ? Est-ce que nous sommes plutôt dans le domaine de l'autofiction ? En fait, nous pouvons ajouter avec un brin de dérision que le média de la bande dessinée ne renouvelle pas vraiment le genre autobiographique, mais le complique davantage. 


\section{La nature de la $\mathrm{BD}$}

La nature minimaliste et humoristique de la bande dessinée n'est pas nécessairement un désavantage par rapport à l'autobiographie. En effet, elle donne à Jung la liberté et la flexibilité d'éviter des détails encombrants, d'où la décision de travailler en noir et blanc et avec le format 170 x $240 \mathrm{~mm}$. Sur le plan narratif, ce choix s'avère être convenable pour représenter la voix innocente et naïve d'un petit garçon.

$\mathrm{Au}$ niveau thématique, ce trait particulier de la bande dessinée est exploité différemment. L'ouvrage de Joseph Witek, Comic Books as History : The Narrative art of Jack Jackson, Art Spiegelman, and Harvey Pekar explore les atouts que procure cette dimension. Dans le cas de Maus, pour ne pas tomber dans le piège du sentimentalisme en abordant le sujet de l'Holocauste, Spiegelman choisit de représenter ses personnages en rats et chats pour se concentrer davantage sur les histoires de son père, survivant des camps de concentration. Cette décision n'affecte pas l'essence du récit et Witek explique que le manque de personnages humains empêche les lecteurs de s'attacher ou de s'identifier aux protagonistes (102-103). La décision artistique de Spiegelman s'applique à Jung, puisque ce dernier laisse libre cours à son imagination grâce aux dimensions humoristiques et minimalistes du média pour recréer les scènes de son enfance et préserver l'innocence enfantine :

Peut-on vraiment parler d'improvisation dans un cadre autobiographique? Mm, disons que c'est une improvisation maîtrisée! Je ne me suis imposé qu'une seule contrainte: éviter toutes formes de misérabilisme ou d'émotion à l'eau de rose. C'est un livre que je veux positif, qu'il soit en quelque sorte à mon image. À la fois sérieux, insouciant, drôle, cynique, optimiste. Je n'aimerais pas que ce livre suscite la pitié, plutôt qu'il touche les gens par son humour, sa charge émotionnelle et son ton décalé. Idéalement, qu'il fasse réfléchir ${ }^{5}$.

Une autre application de cette dimension minimaliste de la BD se matérialise quand Jung présente au public, à priori francophone, la situation sociopolitique en Corée ainsi qu'une vision unique et subjective des conséquences de la guerre, mais toujours sur un ton humoristique. Les explications sur les dimensions sociales et politiques de l'histoire sont réduites au minimum : les cases illustrant l'histoire de la guerre en Corée sont simples, caricaturales et empruntent un style enfantin. Pour montrer l'envergure du problème créé par la guerre, le bédéiste dessine le petit Jung pointant une carte de la France divisée en deux par une ligne pointillée. Sous forme de récitatif, le narrateur pose 
la question: «Pour ceux qui aiment les maths...pas comme moi... voici un petit problème à résoudre: Imaginez la France coupée en 2 parties égales. Combien de familles françaises seraient définitivement séparées ?» (15)

La case suivante ne contient pas de bulles de dialogues mais montre la main d'un petit enfant écrivant «Des millions ». L'aspect enfantin de la bande dessinée crée alors un effet intéressant étant donné qu'il pousse les lecteurs à imaginer les conséquences de la guerre ressenties et perçues par un enfant.

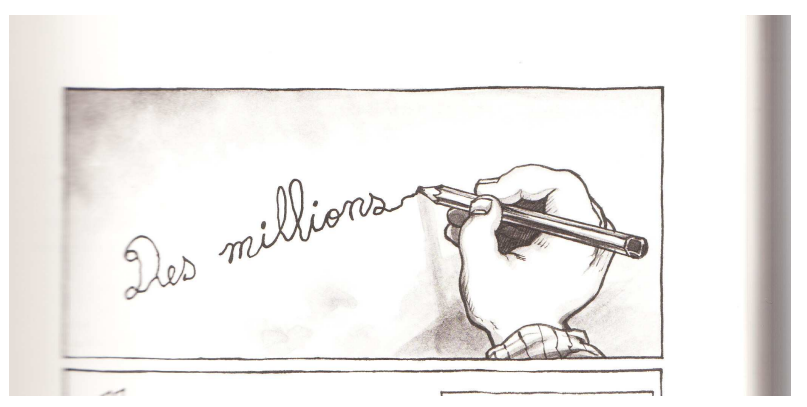

(C) MC Productions / Quadrants / Jung

Dans un monde saturé par les sources médiatiques reportant des pertes humaines comme des chiffres banals, cette case offre une représentation différente qui ne sombre pas dans le sentimentalisme. Nous pouvons ainsi dire que son atout demeure dans la simplicité du message envoyé aux lecteurs.

\section{Le lavis}

La bande dessinée est parfois décrite comme un amalgame de l'écriture et de la peinture. Traditionnellement, une bande dessinée est créé par deux individus, un s'occupant du scénario tandis que l'autre se consacre aux dessins (par exemple Les aventures d'Astérix le gaulois écrit par Goscinny et illustré par Uderzo). Dans le cas de Jung, un seul individu s'approprie la responsabilité des deux tâches. Les bédéistes comme Jung, qui sont à la fois scénaristes et dessinateurs, adoptent souvent un style unique. Toutefois, Couleur de Peau: miel est une œuvre différente des travaux précédents de Jung $^{6}$ considérant le fait que la bande dessinée est en noir et blanc. L'utilisation du noir et blanc dans la bande dessinée autobiographique n'est pas innovateur ${ }^{7}$. Par contre le lavis technique de peinture se limitant à une seule couleur diluée pour obtenir différentes intensités de couleur à l'aquarelle ou à l'encre de Chine - est un choix rare parmi les 
dessinateurs. Dans un entretien, Jung explique ses motivations derrière son choix technique :

Je voulais travailler sur un format A4, papier machine, en noir et blanc avec un traitement en noir et blanc et au lavis. Ce format et cette technique me permettent d'avancer très rapidement, je rentre moins dans les détails, je vais directement à l'essentiel. Ainsi, je peux faire 20 planches toutes les 2 semaines, scénario compris. C'est une manière de travailler très euphorisante, moins laborieuse. J'ai toujours aimé le noir et blanc qui d'ailleurs s'est tout de suite imposé pour ce récit autobiographique. ${ }^{8}$

Le lavis détient également une importance culturelle et identitaire, car elle est une technique très prisée parmi les peintres asiatiques comme l'explique Ernst Grosse :

Si actuellement encore beaucoup d'Extrême-orientaux proclament le lavis la partie la plus noble de leur peinture, aucun de ceux qui connaissent l'Asie orientale moderne, ne se laissera abuser ; ce jugement résulte plutôt du respect dû à une ancienne tradition que d'un amour vivace pour le lavis. (1)

Par ailleurs, nous ne pouvons rester insensibles à la maîtrise de la technique de Jung dans ses magnifiques paysages coréens et dans l'illustration des danseuses coréennes en habits traditionnels. Bien que le choix de lavis repose en premier lieu sur son aspect pratique, le fait d'utiliser une telle technique pour illustrer son autobiographie nous amène à penser que cette décision pourrait être un hommage involontaire aux racines asiatiques de Jung. En somme, le genre autobiographique reçoit ici une innovation non seulement au niveau de l'écriture mais également sur le plan esthétique. À la limite, nous pourrions dire que le lavis est une empreinte laissé par le dessinateur pour évoquer ses origines.

\section{L'espace sonore}

L'espace sonore, c'est-à-dire le contenu des bulles selon Pierre FresnaultDeruelle $^{9}$, est un atout essentiel à l'atmosphère de la bande dessinée. Les dialogues dépouillés, en combinaison avec les images minimalistes, permettent d'arriver directement à l'essentiel.

Un aperçu de l'importance de l'espace sonore se manifeste quand Jung aborde les raisons derrière l'abandon des enfants en Corée. La forme de cette scène, présentée par un narrateur omniscient dans un commentaire d'ordre général, est à la fois imaginée et imitative : une mère en larmes, regardant son enfant dans ses bras avec dans le récitatif : 
«Une maternité hors mariage était une honte, était trop lourde à vivre pour une mère célibataire. Sans argent discréditée par la société, elle n'avait plus qu'une seule solution : l'abandon » (124). Jung imagine ce qu'a dû ressentir la mère au moment de l'abandon. Dans la vignette suivante, il n'y a aucun dialogue mais l'enfant se trouve à côté d'une poubelle remplie d'ordures, avec la mère qui s'éloigne à l'arrière-plan. L'articulation, c'est-à-dire la relation entre les vignettes, attire notre attention sur la violence de la scène grâce à la simplicité de l'espace visuel des deux cases. En comparant l'espace sonore, la deuxième case ignore la narration pour illustrer l'essentiel - l'enfant possède maintenant un air effaré et le fait d'être à proximité d'une poubelle le rabaisse à un objet non désiré et sale. Interposer les deux cases ajoute un effet stylistique qui ne se rapporte pas seulement à l'écriture. Les images fixes de l'art séquentiel nous permettent de visualiser la situation comme une scène entière. L'effet de l'abandon est certainement accentué par la dimension minimaliste du média ; l'approche est directe et efficace.

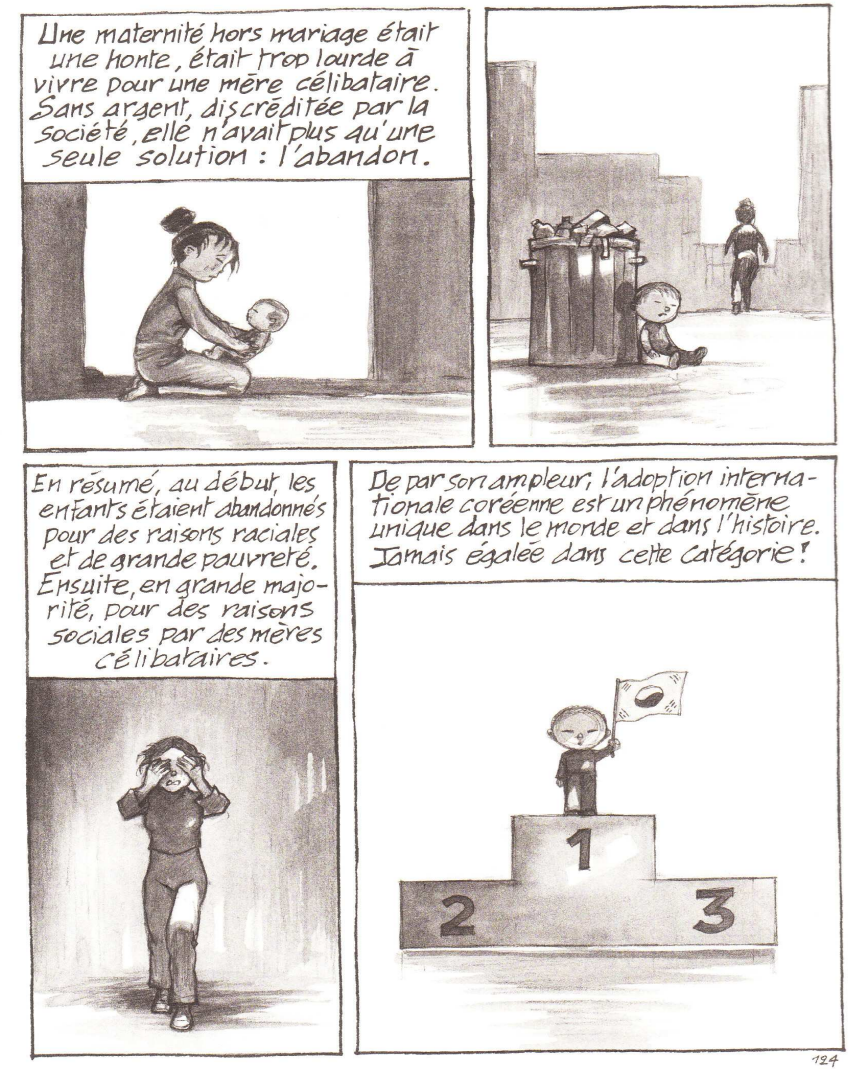

(C) MC Productions / Quadrants / Jung 
Pour rester dans le domaine de l'espace sonore, nous dirons que Couleur de Peau: Miel est innovateur en termes de montage, qui concerne les relations entre les analepses et les prolepses. Cette technique, empruntée au cinéma, n'est pas explorée à fond dans le travail de Jung, mais elle mérite d'être mentionnée. L'interaction entre Jung et l'autre Coréen adopté, commençant à la page 71, est le meilleur exemple des anticipations séquentielles. Les deux enfants affichent du mépris l'un envers l'autre, mais cela est attribué plutôt à un sentiment de honte et d'insécurité selon Jung.

Il imagine alors quelle pourrait être la scène si les personnages abordaient leurs rencontres avec plus de courtoisie. Jung demande alors de «couper» et de refaire la scène, cette fois avec plus de pragmatisme, ce qui échoue lamentablement. En fin de compte, les lecteurs comprennent qu'aucune conversation ne s'est déroulée. Bien que cette technique narrative ne soit pas toujours employée avec succès dans la bande dessinée, le médium possède une versatilité étonnante en ce qui concerne l'emprunt des techniques venant des autres médias telle que l'art du montage.

\section{La bande dessinée comme média pour l'histoire collective}

Couleur de Peau : Miel pose également la question de la relation entre la mémoire individuelle et collective. Dans sa quête d'identité, Jung a recours à sa mémoire pour reconstituer son enfance et son adolescence. Si nous empruntons La mémoire, l'histoire et l'oubli (2003) de Paul Ricœur comme cadre théorique, nous comprenons que la mémoire individuelle contribue à la mémoire collective et qu'elle ne peut exister sans elle. Ainsi, en partageant son récit d'enfance, Jung offre un point de vue unique parmi les «200.000 Coréens adoptés disséminés à travers le monde » (20).

Ann Miller dans Citizenship and City Spaces: Bande dessinée as Reportage suggère que certaines bandes dessinées possèdent un aspect « reporteur ${ }^{10}$. Couleur de Peau: Miel ne fait pas partie de ce genre mais contient des traits spécifiques de cette catégorie car Jung nous invite à nous intéresser au thème de l'adoption. 200000 adoptés est un chiffre énorme et alarmant qui a rarement engendré la curiosité des médias ou des chercheurs. Jung propose d'analyser la question de l'adoption selon ses propres perspectives quand il met en question l'agence d'adoption Holt $^{11}$ ou les raisons pour lesquelles les familles veulent adopter. 
Le fait de donner voix à une portion de la population du monde, inconnue et peu entendue, valorise l'importance de la bande dessinée en termes de critique sociale. Il est sous-entendu que ce média a toujours été relégué au rang de divertissement. Mais Bart Beaty ${ }^{12}$ pense que cette réputation peut tourner à l'avantage des bédéistes. En reprenant le travail de Julia Swindells, il explique que le genre autobiographique, par sa nature émancipatoire, offre aux opprimés une plateforme pour s'exprimer (143). Il faut noter que Beaty est prudent; il ne catégorise pas tous les bédéistes comme des opprimés mais croit que la bande dessinée autobiographique est un mode d'expression puissant s'il est utilisé avec habileté pour aider à représenter un groupe. La bande dessinée de Jung contient ainsi des éléments de reportage et de représentation d'un groupe peu connu, ce qui nous incite à penser que l'autobiographie de Jung s'étend vers un plus grand horizon, incluant la mémoire des autres.

\section{L'altérité}

Déraciné et propulsé subitement dans une société inconnue, l'individu peut vite devenir victime du processus d'intégration. Selon Jung, la solution la plus logique consiste à adopter la culture dominante pour faciliter la transition. Pourtant, il ressent une hantise profonde et une attirance inexplicable envers ses origines, ce qui le mène irrémédiablement à se définir comme un «autre ». On pourrait dire ainsi que l'identité de Jung est affectée et découle de l'altérité, la bande dessinée lui permettant de remettre en cause cette altérité.

Par ironie, Jung est adopté par une famille belge de Wallonie. Le passage qui rend compte de la notion de l'altérité volontaire comprend une série de trois cases montrant Jung en train d'imaginer son identité en Belgique : «L'union fait la force » est la devise des Belges... Ben, c'est mal barré. Et moi, si je suis plus Coréen, je suis flamand, Bruxellois, ou Wallon ? Puis dans la case suivante, le petit garçon choisit la flèche pointant «Wallonie » en disant: «Je ne serai pas Flamand. » La vignette qui suit enchaîne avec le petit garçon choisissant son identité par altérité : «Je ne serai pas Américain, pas Norvégien, pas Italien, pas Français, pas Suédois, pas Allemand, pas Danois, pas Hollandais, pas Anglais. Je serai Belge de Wallonie. Pourquoi pas !» (43). 
La négation par rapport à l'identité est une preuve que Jung se définit plus par ce qu'il n'est pas.

Un autre élément de l'altérité se manifeste dans l'admiration extrême de Jung pour la culture japonaise. Son refus catégorique de ses origines coréennes prend une tournure intéressante lorsqu'il avoue sa difficulté à se débarrasser de ses racines et à s'identifier complètement comme Belge. A vrai dire, sa décision d'adopter la culture japonaise est curieuse mais compréhensible dans le sens où il ne peut nier son identité asiatique :

Il m'arrivait également de dessiner «nos ancêtres les Gaulois ». Fabuleux guerriers, mais si peu «mes ancêtres ». Mes vrais ancêtres, ceux de Corée, je ne voulais pas en entendre parler. J'avais le sentiment qu'ils m'avaient trahi en m'envoyant loin de chez eux, en Europe. A mes yeux, c'était impardonnable. Au tout début de mon adolescence, en tant qu'asiatique, j'ai ressenti le besoin de rattachement à une culture d'Extrême-Orient. De m'identifier à un peuple dont je pouvais être fier. (116)

En se distançant de la Corée volontairement et involontairement, Jung s'identifie comme Belge mais sa fascination pour le Japon remet en cause l'altérité dans sa vie parce qu'il éprouve toujours le besoin de s'attacher à la culture orientale, même si c'est une culture avec laquelle il ne possède aucun lien direct. L'identité de Jung est ainsi croisée avec une altérité à mi-chemin - une identité mixte mais qui ne va pas jusqu'au bout ${ }^{13}$. Malgré ses tentatives de se réinventer, il ne peut échapper à son passé. Mais tout le sujet de l'altérité est aussi une question de perspective : est-ce qu'on perçoit Jung comme Belge ou comme Coréen ? Est-ce que la bande dessinée a été bien reçue parmi les Francophones ? Parmi les Coréens adoptés en Belgique ? Et en Corée du sud ? Puisque la bande dessinée a été traduite en coréen, il serait fort intéressant de suivre la réception et les critiques de l'œuvre de Jung dans son pays natal.

\section{Conclusion}

Lejeune, à propos de la bande dessinée et plus précisément de Maus de Spiegelman dans «Peut-on innover en autobiographie ?», explique que l'originalité associée au média pourrait renouveler le genre autobiographique : «Associer écriture, dessin et peinture pour raconter sa vie : il y a là un champ de possibilités immense (qu'on 
pourrait appeler l'autobio-graphisme...), jusqu'à présent très peu exploré. Une manière comme une autre de créer une forme originale, qui vous ressemble... » (97).

$\mathrm{Si}$ nous suivons la pensée de Lejeune, le simple fait de représenter son autobiographie en forme de bande dessinée permet à la limite d'innover le genre littéraire. Au niveau du contenu, la bande dessinée de Jung explore un sujet peu connu du public et offre une critique et sociale de la question de l'adoption internationale, de sa relation avec la question de l'identité et, finalement, le rôle de l'altérité dans la construction de l'identité raciale. Certes, ces questions ont été déjà abordées dans d'autres contextes et ouvrages, mais nous pouvons supposer que Jung est un des rares écrivains et dessinateurs qui ont présenté l'ensemble de ces sujets dans une bande dessinée. Tout en restant fidèle à la nature simpliste et divertissante de la bande dessinée, Jung transmet son histoire à un public et ouvre une nouvelle porte pour le genre autobiographique ainsi que sa dimension de critique sociale.

\section{Ouvrages cités}

Altarriba, Antonio. «Propositions pour une analyse spécifique de récit en bande dessinée. » Colloque de Cerisy: Bande Dessinée Récit et Modernité. Dirs. Thierry Groensteen. France : Futuropolis. Centre National de la Bande Dessinée et de l'Image, 1988.

Beaty, Bart. Unpopular Culture: Transforming the European Comic Book in the 1990s. Toronto : U of Toronto P, 2007.

Eisner, Will. Comics and Sequential Art: Principles and practices from the legendary cartoonist. New York : Norton, 2008.

Fresnault-Deruelle, Pierre. «L'espace interpersonnel dans les comics ». Sémiologie de la représentation. Dir. André Helbo. France : Complexes. PU France, 1975.

Grosse, Ernst. L'art de l'Orient. Le Lavis en Extrême Orient. Paris : Crès.

Jung. Couleur de peau : miel - Tome 1. Belgique : Quadrants, Astrolabe, 2007.

Lejeune, Philippe. Le pacte autobiographique (1965). Paris : Seuil, 1996.

---. «Peut-on innover en autobiographie?». L'autobiographie. Vles rencontres psychanalytiques d'Aix-en-Provence. Paris : Belles-Lettres, 1988.

Maalouf, Amin. Les identités meurtrières. Paris : Livre de poche, 2001. 
Miller, Ann. «Citizenship and City Spaces : Bande dessinée as Reportage ». History and Politics in French-language Comics and Graphic Novels. Dir. Mark McKinney. Jackson : UP Mississippi, 2008.

Nothomb, Amélie. Métaphysique des tubes. Paris : Albin Michel, 2000.

Ricœur, Paul. Soi-même comme un autre. Paris : Seuil, 1990.

---. La mémoire, l'histoire et l'oubli. Paris : Seuil, 2003.

Saraceni, Mario. The Language of Comics. Londres et New York : Routledge, 2003.

Witek, Joseph. Comic Books as History : The Narrative Art of Jack Jackson, Art Spiegelman, and Harvey Pekar. Jackson : UP Mississippi, 1989.

\section{Site-web consulté}

www.Sceneario.com

\section{NOTES}

${ }^{1}$ La première version fut publiée en 1971 sous la forme d'une bande dessinée de trois pages. Puis Spiegelman décida de prolonger son œuvre. En 1986, apparut le premier volume intitulé, My father bleeds history et en 1991, le deuxième, And here my troubles began.

${ }^{2}$ De son vrai nom, Jung Sik Jun (né en 1965 à Seoul).

${ }^{3}$ Figure hautement respectée dans le milieu de la bande dessinée. Par ailleurs, chaque année, les meilleurs scénaristes et artistes sont couronnés par un Eisner, soit l'équivalent d'un Oscar.

${ }_{5}^{4}$ Le terme « panneau » désigne la page en entier sur laquelle se trouvent les cadres.

${ }^{5}$ Propos tenus par Jung dans son entretien disponible à la fin du tome 1 de la bande dessinée.

${ }^{6}$ À part certains de ses croquis, Jung travaille normalement avec les couleurs. L'écrivain collabore souvent avec sa femme, Jee-Yun, qui est scénariste. Il faut aussi ajouter que les œuvres de Jung s'adressent souvent à un public averti. Au niveau du marketing, Couleur de peau : miel a été lancé et distribué comme bande dessinée pour tout âge.

${ }^{7}$ L'excellente bande dessinée autobiographique Persepolis de Marjane Satrapi, vient à l'esprit.

${ }^{8}$ Propos tenus lors d'un entretien sur le site www.sceneario.com.

${ }^{9}$ Fresnault-Deruelle, Pierre. L'espace interpersonnel dans les comics. Sémiologie de la représentation. Dir. André Helbo. Paris : Complexes. PUF, 1975.

${ }^{10}$ Selon Miller, la référence par excellence se manifeste sous le personnage de Tintin, héros mythique d'Hergé.

${ }^{11}$ Holt International est une organisation d'adoption charitable établie par Harry et Bertha Holt en 1955, dont la mission principale consiste à accueillir les orphelins et à les aider à trouver des familles d'adoption. Holt International a une forte présence en Corée du sud étant donné que le but initial était de s'occuper des orphelins durant la guerre de Corée. Voir www.holtinternational.org

${ }^{12}$ Beaty, Bart. Unpopular Culture: Transforming the European Comic Book in the 1990s. Toronto : U of Toronto P, 2007.

${ }^{13}$ Concernant les identités croisées avec une altérité à mi-chemin, nous pouvons certainement mentionner la prolifique écrivaine belge, Amélie Nothomb, qui a longtemps travaillé sur le genre autobiographique. Née d'une famille belge, Nothomb a vécu et grandi en Asie, notamment au Japon. Métaphysique des tubes (2000) et Stupeur et tremblements (1999) sont deux textes autobiographiques qui abordent la question de l'identité et de l'intégration dans une société dominante pour un individu à identités multiples. 